\title{
Femoral Neck
}

National Cancer Institute

\section{Source}

National Cancer Institute. Femoral Neck. NCI Thesaurus. Code C61563.

The short, constricted portion of the thigh bone between the femur head and the trochanter. 